\title{
European boost for particle therapy
}

Treatment centres poised to use carbon-ion beams to tackle cancer.

The first European clinical centre offering carbon-ion therapy to treat cancer is set to open early this year in Heidelberg, Germany, followed by a second facility in Pavia, Italy, by 2010 .

Proponents of the technique - which uses beams of ions to kill tumour cells - say the new centres mark a significant watershed for a field that has been slowly gaining ground for more than 60 years.

In 1946, Robert Wilson, a physicist on the Manhattan Project and later head of the Fermi National Accelerator Laboratory in Illinois, proposed using charged particles to treat cancer. Since then, there has been growing evidence that beams of protons and carbon ions known collectively as hadron therapy - may offer an important alternative to X-rays for treating cancer.

Unlike X-rays, which deposit most of their energy near the skin's surface, hadron beams are more effective at reaching tumour cells in deeper tissue. The particles release the bulk of their energy as they slow down in the last few millimetres of their journey, a point called the Bragg peak. The beams also scatter very little, allowing the maximum radiation dose to be precisely targeted to tumour cells, and thus minimizing damage to surrounding healthy tissue, explains biologist Manjit Dosanjh, director of the European Network for Research in Light Ion Hadron Therapy at CERN.

Neutron beams share many of these properties but are more difficult to guide because of their lack of charge, necessitating higher doses that can cause severe side effects. Today, only a handful of clinics offer neutron therapy.

Proton therapy is in regular use at medical centres around the world, whereas carbon-ion therapy is at a much more experimental stage. But researchers believe that it may offer even greater accuracy in targeting tumour cells, which, combined with carbon ions' greater mass, gives the beams a much sharper Bragg peak.

Carbon ions also produce a different type of cellular damage from protons or photons, explains Ugo Amaldi, a medical physicist at the University of Milan-Bicocca, Italy, and CERN, the European particle-physics centre near Geneva in Switzerland. "Conventional radiation damages DNA indirectly through the production of free radicals, which requires oxygen to work," says Amaldi. Carbon ions directly cleave double-stranded DNA at multiple sites without the need for oxygen, so they can tackle hypoxic areas of tumours that are resistant to radiotherapy. "About $10 \%$ of all tumours are resistant to X-rays and protons," says Amaldi. "Such tumours - in the brain, liver and lung - have a greater chance of responding to bombardment with carbon ions."

Japan, a pioneer in the field, began testing carbon-ion therapy in 1994, and opened the world's first dual-therapy facility at the Hyogo

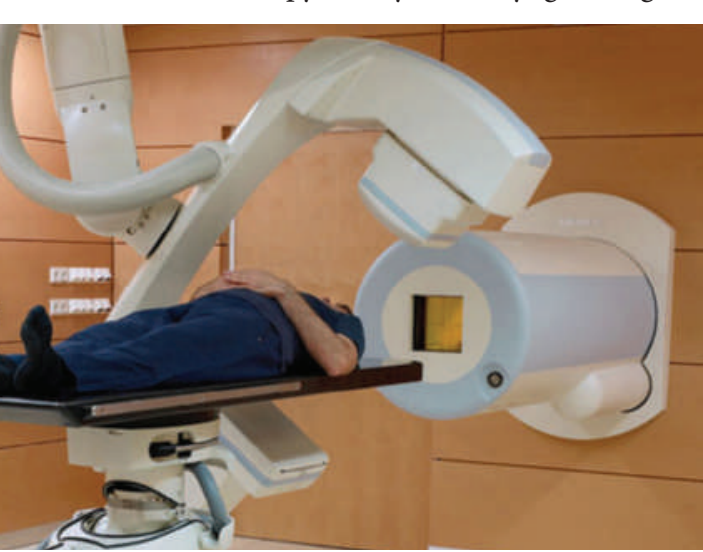
is planned for the near future in the United States: at Touro University near San Francisco, California. The facility expects to have its $\$ 75$-million proton accelerator in place by 2010 - its carbon-ion capability will follow by 2012 at an additional cost of $\$ 121$ million. But most cancer centres - and the National Institutes of Health - say that they are not ready to invest in expensive carbon-ion-therapy centres without further clinical data.

"Carbon-ion beams may have an advantage in certain tumour types, but that has not yet been proven," says Herman Suit, chief of radiation oncology at the Dana-Farber/Harvard Cancer Center in Boston, Massachusetts, who has conducted proton-therapy research since 1973. Even if carbon ions damage tumours more effectively, he says, it is an open question whether they cause longer-term problems, such as triggering the formation of secondary tumours.

In contrast to the situation in the United States, carbon-ion therapy is attracting growing funding in Europe. Half of the HIT's start-up funding has come from a German government loan, whereas the
Ion Beam Medical Center in 2001. A pilot carbon-ion therapy centre at the Society for Heavy Ion Research (GSI) in Darmstadt, Germany, has treated about 450 patients since 1997.

But critics say that there are not enough data to show that carbon-ion therapy is any more effective than conventional treatments. A systematic literature review in 2007 , led by the Cochrane Cancer Network, based in Oxford, UK, argued that although early results have been promising, most studies have been done in physics facilities, which did not run randomized, controlled clinical trials (M. Lodge et al. Radiother. Oncol. 83, 110-122; 2007).

It is hoped that the two new facilities the Heidelberg Ion Therapy Centre (HIT) and Pavia's National Centre for Oncological Hadron Therapy (CNAO) - will rectify that deficit with much more extensive clinical trials. The facilities aim to compare protons and carbon ions, used separately and in combination, with X-rays and surgery, and will be the first in Europe to offer both therapies to treat a range of tumours, including spinal-cord and ocular tumours, and lung, prostate and liver cancer.

Only one comparable dual-particle facility creation of the CNAO will be funded entirely by the Italian government. Five other European carbon-ion or dual-particle centres are planned: in Wiener Neustadt, Austria; Lyon, France; Marburg and Kiel in Germany; and Stockholm. Like the HIT, the two German facilities will be operated by Siemens, the engineering conglomerate.

The field still faces significant hurdles, though. The HIT's accelerator system has been ready for more than six months, says Thomas Haberer, the centre's scientific director, but problems with the software that controls the ion beams have delayed the centre's opening. And researchers at both the HIT and CNAO expect that it will take years to refine their beam-scanning techniques to allow them to treat highly mobile organs such as the lungs.

But pioneers in ion therapy are bullish. "Europe is at the frontier of this research," says Gerhard Kraft, who oversaw the GSI's iontherapy programme for many years before his retirement. "Carbon ions are sometimes considered potentially dangerous - but that's because they are extremely effective."

Vicki Brower 